\title{
Spectral Families of Quantum Stochastic Integrals
}

\author{
David Applebaum \\ Department of Mathematics, Statistics and Operational Research, The Nottingham Trent University, \\ Burton Street, Nottingham. NG1 4BU, England
}

Received: 28 March 1994 / in revised form: 14 October 1994

\begin{abstract}
We develop a theory of spectral integration for quantum stochastic integrals of certain families of processes driven by creation, conservation and annihilation processes in Fock space. These give a non-commutative generalisation of classical stochastic integrals driven by Poisson random measures. A stochastic calculus for these processes is developed and used to obtain unitary operator valued solutions of stochastic differential equations. As an application we construct stochastic flows on operator algebras driven by Lévy processes with finite Lévy measure.
\end{abstract}

\section{Introduction}

The standard theory of quantum stochastic calculus in boson Fock space as developed by R.L. Hudson and K.R. Parthasarathy uses the creation, conservation and annihilation processes as basic martingales (see [HuPa 1], [Par], [Mey] and references therein) and it is well known that classical stochastic integrals with respect to a standard Brownian motion or compensated Poisson process can be constructed within this more general formalism. As it stands however the theory is insufficiently fine to capture stochastic integrals with respect to Poisson random measures. The aim of this paper is to take the first steps towards developing the required generalisation.

Of course stochastic integrals with respect to Poisson random measures involve two distinct integrations over both time and space variables. In the quantum case we find that integration over the "space" variable corresponds to a spectral integral and hence the objects which we need to make sense of are operators of the form

$$
\begin{gathered}
M(t)=\int_{-\infty}^{\infty} \int_{0}^{t}\left\{E_{1}(s, \lambda) A_{x}^{\dagger}(d s, P(d \lambda))+E_{2}(s, \lambda) \Lambda(d s, P(d \lambda))\right. \\
\left.+E_{3}(s, \lambda) A_{y}(d s, P(d \lambda))\right\}
\end{gathered}
$$

where $P$ is a projection-valued measure, $E_{j}(j=1,2,3)$ are suitable families of adapted processes and $A^{\dagger}, \Lambda$ and $A$ represent the differentials of the creation, conservation and annihilation processes (respectively) in a sense which is made precise 
below. From the point of view that regards quantum stochastic integration as describing the coupling of a quantised system to a heat bath, it seems clear that the finer decomposition given in (1.1) yields more information about the coupling than the standard integral.

Integrals of the form (1.1) first appeared in [App 1] where they appeared naturally in the construction of new types of quantum stochastic flow from classical Lévy flows, however in [App 1] they were only defined as forms and a full stochastic calculus for them was not developed as is the case below. We remark that a similar class of integrals has recently been employed by Belavkin [Bel] in his work on the quantum measurement problem.

The organisation of this paper is as follows. In Sect. 2 we collect some preliminary facts about quantum stochastic integrals which we will use in the development of our theory. In Sect. 3, we construct the integrals (1.1) as limits of sums of stochastic integrals defined over partitions. We obtain an Itô formula for the product of two such processes. Sect. 4 is devoted to proving a Fubini type theorem in which it is demonstrated that the two integrals in (1.1) can be interchanged. Our processes are used in Sect. 5 to construct solutions of linear stochastic differential equations (SDE's), conditions are established for the unitarity of such solutions and the resulting quantum flow is used to construct a new type of stochastic dilation for norm continuous quantum dynamical semigroups. Finally in Sect. 6, we use Parthasarathy's representation of Lévy processes in Fock space to construct a class of one-dimensional Lévy flows on *-subalgebras of $\mathrm{B}\left(\mathfrak{H}_{0}\right)$ in the case where the Lévy process has finite Lévy measure. Although our method is sufficiently robust for us to drop this latter restriction, there are a number of lengthy technicalities which arise in the treatment of general Lévy measures, hence to avoid this paper becoming of unmanageable length we have postponed the case of general Lévy flows to a later article.

There are a number of interesting questions relating to the integrals (1.1) which are not addressed in this paper. One of the most intriguing of these arises from the observation that the processes given by (1.1) are martingales which suggest the possibility of improving on the martingale representation theorems of [PaSi].

Notation: If $\mathfrak{D}_{i}$ are dense linear manifolds in complex Hilbert spaces $\mathfrak{H}_{i}(i=1,2)$, we denote by $\mathfrak{D}_{1} \otimes \mathfrak{D}_{2}$, their algebraic tensor product. If $L \in B\left(\mathfrak{H}_{1}\right)$ we often write its ampliation $L \otimes I$ to the whole of $\mathfrak{H}_{1} \otimes \mathfrak{H}_{2}$ simply as $L$ provided there is no room for ambiguity. If $\mathfrak{I}$ is a topological space, $\mathscr{B}(\mathfrak{I})$ will denote the $\sigma$-algebra of all Borel sets in $\mathfrak{I}$.

\section{Preliminaries (cf.[HuPa 1], [Pa])}

Let $\mathfrak{H}_{0}$ and $\mathfrak{H}_{1}$ be complex separable Hilbert spaces and let $\mathfrak{H}$ denote the Hilbert space tensor product $\mathfrak{H}_{0} \otimes \Gamma(\mathscr{H})$, where $\mathscr{H}=L^{2}\left(\mathbb{R}^{+}, \mathfrak{H}_{1}\right)$. For $f \in \mathscr{H}$, the exponential vector $\psi(f) \in \Gamma(\mathscr{H})$ is defined by

$$
\psi(f)=\left(1, f, \frac{f \otimes f}{\sqrt{2 !}}, \cdots \frac{f^{\otimes n}}{\sqrt{n !}}, \cdots\right)
$$

so that if $g \in \mathscr{H}$ then $\langle\psi(f), \psi(g)\rangle=\exp (\langle f, g\rangle)$. 
Let $\mathscr{S}$ be a dense linear manifold in $\mathscr{H}$ and let $\mathscr{E}(\mathscr{S})$ denote the linear span of $\{\psi(f), f \in \mathscr{S}\}$, then $\mathscr{E}(\mathscr{S})$ is itself dense in $\Gamma(\mathscr{H})$. Throughout this paper we will take $\mathscr{S}$ to consist of $\mathfrak{H}_{1}$-valued locally bounded functions on $\mathbb{R}^{+}$.

For $f, g \in \mathscr{H}$ and $T \in B(\mathscr{H})$ with $\mathrm{T}=\mathrm{T}^{*}$, we denote by $a^{\dagger}(f), \lambda(T)$ and $a(g)$ respectively the corresponding creation, conservation and annihilation operators in $\Gamma(\mathscr{H})$.

For the remainder of this section we fix once and for all $x, y \in \mathfrak{H}_{1}$ and $H \in B\left(\mathfrak{S}_{1}\right)$ with $H=H^{*}$. We define the creation process $A_{x}^{\dagger}=\left(A_{x}^{\dagger}(t), t \in \mathbb{R}^{+}\right)$ by

$$
A_{x}^{\dagger}(t)=a^{\dagger}\left(\chi_{[0, t)} \otimes x\right)
$$

the conservation process $\Lambda_{H}(t)=\left(\Lambda_{H}(t), t \in \mathbb{R}^{+}\right)$by

$$
\Lambda_{H}(t)=\lambda\left(\chi_{[0, t)} \otimes H\right)
$$

and the annihilation process $A_{y}(t)=\left(A_{y}(t), t \in \mathbb{R}^{+}\right)$by

$$
A_{y}(t)=a\left(\chi_{[0, t)} \otimes y\right) .
$$

We note that

$$
\mathscr{E}(\mathscr{S}) \subset \operatorname{Dom}\left(A_{x}^{\dagger}(t)\right) \cap \operatorname{Dom}\left(\Lambda_{H}(t)\right) \cap \operatorname{Dom}\left(A_{y}(t)\right)
$$

and

$$
A_{x}^{\dagger}(t) \subset A_{x}(t)^{*}, \quad \Lambda_{H}(t) \subset \Lambda_{H}(t)^{*}, \quad \text { for all } t \in \mathbb{R}^{+} .
$$

Recall that, for each $t \in \mathbb{R}^{+}, \mathfrak{H}$ may be identified with the Hilbert space tensor product $\mathfrak{S}_{t} \otimes \mathfrak{S}^{t}$, where $\mathfrak{H}_{t}=\mathfrak{H}_{0} \otimes \Gamma\left(L^{2}\left([0, t), \mathfrak{H}_{1}\right)\right)$ and $\mathfrak{H}^{t}=\Gamma\left(L^{2}\left([t, \infty), \mathfrak{H}_{1}\right)\right)$ by means of the canonical isomorphism which maps each $u \otimes \psi(f)$ to $(u \otimes$ $\psi\left(f \chi_{[0, t)}\right) \otimes \psi\left(f \chi_{[t, \infty)}\right)$, where $u \in \mathfrak{H}_{0}, f \in \mathscr{H}$.

We fix a dense linear manifold $\mathfrak{D}_{0}$ in $\mathfrak{H}_{0}$.

A family $E=\left(E(t), t \in \mathbb{R}^{+}\right)$of densely defined linear operators in $\mathfrak{H}$ is said to be adapted if

(i) $\mathfrak{D}_{0} \otimes \mathscr{E}(\mathscr{S}) \subseteq \operatorname{Dom}(E(t))$.

(ii) Each $E(t)=\hat{\hat{E}}(t) \otimes I^{t}$, where $\hat{E}(t)$ is an operator in $\mathfrak{G}_{t}$ and $I^{t}$ is the identity in $B\left(\mathfrak{H}^{t}\right)$.

Let $\mathscr{P}=\left\{0=t_{0}<t_{1}<\ldots<t_{n} \rightarrow \infty\right\}$ be a partition of $\mathbb{R}^{+}$. An adapted process $E$ is simple with respect to $\mathscr{P}$ if

$$
E(t)=E\left(t_{J}\right) \quad \text { whenever } t_{J} \leqq t<t_{j+1} .
$$

We denote the class of all simple processes by $\Sigma\left(\mathfrak{D}_{0}, \mathscr{S}\right)$.

An adapted process $E$ is locally square integrable if for each $u \in \mathfrak{H}_{0}, f \in \mathscr{S}$ the map $t \rightarrow E(t)(u \otimes \psi(f))$ is measurable and for each $t>0$,

$$
\int_{0}^{t}\|E(s)(u \otimes \psi(f))\|^{2} d s<\infty .
$$

The linear space of all such processes will be denoted $L_{\text {loc }}^{2}\left(\mathfrak{D}_{0}, \mathscr{S}\right)$. 
It is proved in $[\mathrm{Ev}]$ that given any $E \in L_{\text {loc }}^{2}\left(\mathfrak{D}_{0}, \mathscr{S}\right)$, there exists a sequence $\left(E_{n}\right)_{n \in \mathbb{N}}$ in $\Sigma\left(\mathfrak{D}_{0}, \mathscr{S}\right)$ such that for all $t \in \mathbb{R}^{+}, u \in \mathfrak{D}_{0}, f \in \mathscr{S}$,

$$
\int_{0}^{t}\left\|\left(E(s)-E_{n}(s)\right)(u \otimes \psi(f))\right\|^{2} d s \underset{n}{\longrightarrow} 0 .
$$

Let $\left(E_{j}, j=1,2,3,4\right)$ be simple processes and assume (without loss of generality) that they are defined with respect to a common partition $\mathscr{P}$. Their stochastic integral $M=\left(M(t), t \in \mathbb{R}^{+}\right)$with respect to the processes $A_{x}^{\dagger}, \Lambda_{H}, A_{y}$ and "time" is the adapted process given by

$$
\begin{aligned}
M(t)=\sum_{n=0}^{\infty} & \left\{E_{1}\left(t_{n} \wedge t\right) a^{\dagger}\left(\chi_{\left[t_{n} \wedge t, t_{n+1} \wedge t\right)} \otimes x\right)+E_{2}\left(t_{n} \wedge t\right) \lambda\left(\chi_{\left[t_{n} \wedge t, t_{n+1} \wedge t\right)} \otimes H\right)\right. \\
& \left.+E_{3}\left(t_{n} \wedge t\right) a\left(\chi_{\left[t_{n} \wedge t, t_{n+1} \wedge t\right)} \otimes y\right)+E_{4}\left(t_{n} \wedge t\right)\left(t_{n+1} \wedge t-t_{n} \wedge t\right)\right\} .
\end{aligned}
$$

$M$ is usually written in the "integral notation" as

$$
M(t)=\int_{0}^{t}\left(E_{1}(s) d A_{x}^{\dagger}(s)+E_{2}(s) d \Lambda_{H}(s)+E_{3}(s) d A_{y}(s)+E_{4}(s) d s\right),
$$

or the "differential notation" as

$$
d M=E_{1} d A_{x}^{\dagger}+E_{2} d \Lambda_{H}+E_{3} d A_{y}+E_{4} d t .
$$

We will occasionally use an alternative notation of $d A(t, x)$ in place of $d A_{x}(t)$ (etc.) when $x$ is a complicated expression and the above notation is too clumsy.

For $u, v \in \mathfrak{H}_{0}, f, g \in \mathscr{S}$ we obtain the following formulae which will be used extensively in the sequel:

$$
\begin{aligned}
\langle u & \otimes \psi(f), M(t)(v \otimes \psi(g))\rangle \\
& =\int_{0}^{t}\langle u \otimes \psi(f), K(f, g, x, H, y ; s)(v \otimes \psi(g))\rangle d s
\end{aligned}
$$

for each $t \in \mathbb{R}^{+}$, where

$$
K(f, g, x, H, y ; s)=(f(s), x) E_{1}(s)+(f(s), H g(s)) E_{2}(s)+(y, g(s)) E_{3}(s)+E_{4}(s)
$$

and $(.,$.$) is the inner product in \mathfrak{H}_{1}$.

If $M^{j}$ is a stochastic integral of the type (2.2) of a quadruple of simple processes $\left(E_{k}^{j}, k=1,2,3,4\right)$ for $j=1,2$ (respectively), we have

$$
\begin{aligned}
& \left\langle M^{1}(t)(u \otimes \psi(f)), M^{2}(t)(v \otimes \psi(g))\right\rangle \\
& =\int_{0}^{t}\left\{\left\langle M^{1}(s)(u \otimes \psi(f)), K^{2}(f, g, x, H, y ; s)(v \otimes \psi(g))\right\rangle\right. \\
& \quad+\left\langle K^{1}(g, f, x, H, y ; s)(u \otimes \psi(f)), M^{2}(s)(v \otimes \psi(g))\right\rangle \\
& \left.\quad+\left\langle\left\langle L^{1}(f, x, H ; s)(u \otimes \psi(f)), L^{2}(g, x, H ; s)(v \otimes \psi(g))\right\rangle\right\rangle\right\} d s
\end{aligned}
$$

for each $t \in \mathbb{R}^{+}$, where for each $h \in \mathscr{S}, j=1,2$

$$
L^{j}(h, x, H ; s)=x \otimes E_{1}^{J}(s)+H h(s) \otimes E_{2}^{j}(s)
$$


(so that each such $L^{j}$ is a linear operator from $\mathfrak{H}$ into $\mathfrak{H}_{1} \otimes \mathfrak{H}$ ) and $\langle\langle.,\rangle$.$\rangle denotes$ the inner product in $\mathfrak{H}_{1} \otimes \mathfrak{H}$.

Writing $M=M^{1}=M^{2}, g=f$ and $v=u$ in (2.6), we obtain the following estimate for $0 \leqq t \leqq T$,

where

$$
\|M(t)(u \otimes \psi(f))\|^{2} \leqq \int_{0}^{T} e^{t-s} \phi(s) \alpha(x, H, y ; f, s) d s,
$$

$$
\begin{gathered}
\alpha(x, H, y ; f, t)=6 \max \left\{|(f(t), x)|^{2},|(f(t), H f(t))|^{2},|(y, f(t))|^{2},\|H f(t)\|_{\mathfrak{S}_{1}}^{4},\|x\|_{\mathfrak{S}_{1}}^{4}\right\} \\
\text { and } \phi(s)=\sum_{j=1}^{4}\left\|E_{j}(s)(u \otimes \psi(f))\right\|^{2} .
\end{gathered}
$$

For the remainder of this section we take $E_{4}=0$. Now let $P$ be a projection in $\mathfrak{H}_{1}$. By using estimates of the form $|(x, P y)| \leqq\|P x\| .\|P y\| \leqq \frac{1}{2}\left(\|P x\|^{2}+\|P y\|^{2}\right)$, and the Hilbert space inequality $\left\|\phi_{1}+\phi_{2}+\phi_{3}\right\| \leqq\left[3\left(\phi_{1}^{2}+\phi_{2}^{2}+\phi_{3}^{2}\right)\right]^{1 / 2}$, we obtain

$$
\|K(f, f, P x, P, P y ; s)\| \leqq 2(3 \phi(s))^{1 / 2} \beta(x, y, f, P ; s),
$$

where

$$
\beta(x, y, f, P ; s)=\|P x\|^{2}+\|P y\|^{2}+\|P f(s)\|^{2} .
$$

A similar argument establishes the estimate

$$
\|\mid L(f, x, P ; s)(u \otimes \psi(f))\| \|^{2} \leqq 2 \phi(s) \gamma(x, f, P ; s)
$$

where ||$|\cdot|||$ denotes the norm in $\mathfrak{H}_{1} \otimes \mathfrak{H}$ (we have suppressed the index $j$ with obvious meaning) and

$$
\gamma(x, f, P ; s)=\|P x\|^{2}+\|P f(s)\|^{2} .
$$

We will find (2.8) and (2.10) of use in the next section.

Now let $\left(M^{n}\right)_{n \in \mathbb{N}}$ be a sequence of stochastic integrals of the form (2.3) wherein each $\left(E_{j}^{n}\right)_{n \in \mathbb{N}}$ converges to $E_{j} \in L_{\text {loc }}^{2}\left(\mathfrak{D}_{0}, \mathscr{S}\right)$ in the sense of (2.1). Using (2.7) it can be shown that $\left(M^{n}\right)_{n \in \mathbb{N}}$ converges (in an appropriate strong sense) to yield an adapted process $M$ which we call the stochastic integral of the quadruple $\left(E_{j}, j=\right.$ $1,2,3,4)$.

Furthermore the formulae (2.5) to (2.11) remain valid for these integrals. We continue to use the notation (2.3) and (2.4) for such processes.

The following result will be useful in the next section

Lemma 2.1. Let $x_{j}, y_{j} \in \mathfrak{H}_{1}$ and $H_{j} \in B\left(\mathfrak{H}_{1}\right)$ with $H_{j}=H_{j}^{*}, j=1,2$ and let $E_{j} \in$ $L_{\text {loc }}^{2}\left(\mathfrak{D}_{0}, \mathscr{S}\right), j=1,2,3$, then for each $t \in \mathbb{R}^{+}$,

$$
\begin{aligned}
& \int_{0}^{t}\left(E_{1}(s) d A_{x_{1}+x_{2}}^{\dagger}(s)+E_{2}(s) d \Lambda_{H_{1}+H_{2}}(s)+E_{3}(s) d A_{y_{1}+y_{2}}(s)\right) \\
& =\int_{0}^{t}\left(E_{1}(s) d A_{x_{1}}^{\dagger}(s)+E_{2}(s) d \Lambda_{H_{1}}(s)+E_{3}(s) d A_{y_{1}}(s)\right) \\
& \quad+\int_{0}^{t}\left(E_{1}(s) d A_{x_{2}}^{\dagger}(s)+E_{2}(s) d \Lambda_{H_{2}}(s)+E_{3}(s) d A_{y_{2}}(s)\right) .
\end{aligned}
$$

The proof is straightforward and hence is omitted. 


\section{Construction of Quantum Stochastic Spectral Integrals}

For the remainder of this paper we will assume that $\mathfrak{H}_{1}$ is infinite dimensional. Let $Y$ be a (not necessarily bounded) self adjoint operator in $\mathfrak{H}_{1}$ with spectral decomposition $\int_{-\infty}^{\infty} \lambda P(d \lambda)$. Our goal in this section is to make sense, as linear operators in $\mathfrak{H}$, of expressions of the form,

$$
\begin{gathered}
M(t)=\int_{-\infty}^{\infty} \int_{0}^{t}\left\{E_{1}(s, \lambda) A_{x}^{\dagger}(d s, P(d \lambda))+E_{2}(s, \lambda) \Lambda(d s, P(d \lambda))\right. \\
\left.+E_{3}(s, \lambda) A_{y}(d s, P(d \lambda))\right\}
\end{gathered}
$$

where each $\left\{E_{j}(\cdot, \lambda),-\infty<\lambda<\infty\right\}$ for $j=1,2,3$ is a suitable family of processes in $L_{\text {loc }}^{2}\left(\mathfrak{D}_{0}, \mathscr{S}\right)$.

Operators of the form (3.1) will be called quantum spectral stochastic integrals.

Note first of all that if $Y$ has discrete spectrum $\left\{\lambda_{1}<\lambda_{2}<\cdots<\lambda_{n}\right\}$ then we can define $M(t)$ by

$$
\begin{gathered}
M(t)=\sum_{j=1}^{n} \int_{0}^{t}\left\{E_{1}\left(s, \lambda_{j}\right) d A_{P_{j} x}^{\dagger}(s)+E_{2}\left(s, \lambda_{j}\right) d \Lambda_{P_{j}}(s)\right. \\
\left.+E_{3}\left(s, \lambda_{j}\right) d A_{P_{,} y}(s)\right\}
\end{gathered}
$$

where $P_{J}=P\left(\left\{\lambda_{J}\right\}\right)$ for $1 \leqq j \leqq n$.

From now on we will assume that the spectrum of $Y$ is the whole real line. Our strategy will be to obtain (3.1) as a limit of expressions of the form (3.2). First though we will need to make some restrictions on those families of processes which we will be able to integrate. Let $E=\{E(\lambda), \lambda \in \mathbb{R}\}$ be a family of adapted processes in $\mathfrak{H}$ so that each $E(\lambda)=\left(E(t, \lambda), t \in \mathbb{R}^{+}\right)$. Let $[a, b]$ be a fixed closed interval. A family $E$ will be said to be $\lambda$-continuous on $[a, b]$ if for all $t \in \mathbb{R}^{+}, u \in$ $\mathfrak{D}_{0}, f \in \mathscr{S}$, it is the case that given any $\varepsilon>0$, there exists $\delta>0$ such that for any $a \leqq \lambda, \mu \leqq b$ with $|\lambda-\mu|<\delta$, then

$$
\sup _{0 \leqq s \leqq t}\|(E(s, \lambda)-E(s, \mu))(u \otimes \psi(f))\|<\varepsilon .
$$

We note that if $E$ is $\lambda$-continuous on $[a, b]$, then the map

$$
\lambda \rightarrow \sup _{0 \leqq s \leqq t}\|E(s, \lambda)(u \otimes \psi(f))\| \text { is continuous from }[a, b] \text { to } \mathbb{R}^{+} \text {. }
$$

A family $E$ is said to be admissible on $[a, b]$ if

(i) $E(\lambda) \in L_{\mathrm{loc}}^{2}\left(\mathfrak{D}_{0}, \mathscr{S}\right)$ for each $a \leqq \lambda \leqq b$,

(ii) $E$ is $\lambda$-continuous on $[a, b]$.

We will now construct the integrals (3.1). Let $\mathscr{P}=\left\{a=\lambda_{0}<\lambda_{1}<\ldots<\right.$ $\left.\lambda_{n+1}=b\right\}$ be a partition of $[a, b]$. We define the mesh $|\mathscr{P}|$ of $\mathscr{P}$ by $|\mathscr{P}|=$ $\max _{0 \leqq j \leqq n}\left|\lambda_{j+1}-\lambda_{j}\right|$. 
Let $\left\{E_{J}, j=1,2,3\right\}$ be admissible families and define for each $t \in \mathbb{R}^{+}, u \in$ $\mathfrak{D}_{0}, f \in \mathscr{S}$,

$$
\begin{gathered}
\gamma_{t}(\mathscr{P}, u, f)=\sup \left\{\sum_{j=1}^{3} \sup _{0 \leqq s \leqq t}\left\|\left(E_{j}(s, \lambda)-E_{j}(s, \mu)\right)(u \otimes \psi(f))\right\|^{2},\right. \\
a \leqq \lambda<\mu \leqq b,|\lambda-\mu| \leqq|\mathscr{P}|\}^{1 / 2} .
\end{gathered}
$$

Now let $\mathscr{P}^{\prime}$ be a refinement of $\mathscr{P}$ and for each $0 \leqq j \leqq n$, let $\lambda_{J_{1}}, \ldots, \lambda_{J_{n_{j}}}$ be the points of $\mathscr{P}^{\prime}$ which lie between $\lambda_{j}$ and $\lambda_{J+1}$. Define for each $t \in \mathbb{R}^{+}$,

$$
\begin{aligned}
M_{\mathscr{P}}(t)=\sum_{j=0}^{n} \int_{0}^{t}\left\{E_{1}\left(s, \mu_{j}\right) d A^{\dagger}\left(s, P\left(\lambda_{j}, \lambda_{j}\right) x\right)\right. \\
\left.\quad+E_{2}\left(s, \mu_{j}\right) d \Lambda\left(s, P\left(\lambda_{j}, \lambda_{j+1}\right)\right)+E_{3}\left(s, \mu_{j}\right) d A\left(s, P\left(\lambda_{j}, \lambda_{j+1}\right) y\right)\right\},
\end{aligned}
$$

where each $\lambda_{J} \leqq \mu_{J} \leqq \lambda_{J+1}$ for $0 \leqq j \leqq n$.

Lemma 3.1. For each $u \in \mathfrak{H}_{0}, f \in \mathscr{S}$ and $T \in \mathbb{R}^{+}$, there exists a constant $C(T)$ $\geqq 0$ such that for $0 \leqq t \leqq T$,

$$
\left\|\left(M_{\mathscr{P}}(t)-M_{\mathscr{P P}}(t)\right)(u \otimes \psi(f))\right\| \leqq C(T) \gamma_{T}(u, f ; \mathscr{P}) .
$$

Proof. As $P$ is a projection-valued measure, we have that for each $0 \leqq j \leqq n$,

$$
P\left(\lambda_{j}, \lambda_{j+1}\right)=\sum_{l=0}^{n_{j}} P\left(\lambda_{j_{l}}, \lambda_{j_{l+1}}\right)
$$

Hence by Lemma 2.1, we obtain

$$
\begin{aligned}
M_{\mathscr{P}}(t)-M_{\mathscr{P}}(t)=\sum_{j=0}^{n} \sum_{l=0}^{n_{l}} \int_{0}^{t}\{ & \left(E_{1}\left(s, \mu_{J}\right)-E_{1}\left(s, \mu_{j_{l}}\right)\right) d A^{\dagger}\left(s, P\left(\lambda_{j_{l}}, \lambda_{j_{l+1}}\right) x\right) \\
& +\left(E_{2}\left(s, \mu_{j}\right)-E_{2}\left(s, \mu_{J_{l}}\right)\right) d \Lambda\left(s, P\left(\lambda_{j_{l}}, \lambda_{J_{l+1}}\right)\right) \\
& \left.+\left(E_{3}\left(s, \mu_{j}\right)-E_{3}\left(s, \mu_{j_{l}}\right)\right) d A\left(s, P\left(\lambda_{j_{l}}, \lambda_{j_{l+1}}\right) y\right)\right\} .
\end{aligned}
$$

We will write each $P\left(\Delta_{J_{l}}\right)=P\left(\lambda_{j_{l}}, \lambda_{j_{l+1}}\right)$, for simplicity. find

Now by (2.6) and a similar argument to that of Corollary 1 of $[\mathrm{HuPa} 1]$, we

$$
\begin{gathered}
\left\|\left(M_{\mathscr{P}}(t)-M_{\mathscr{P} \prime}(t)\right)(u \otimes \psi(f))\right\|^{2} \\
=\left\langle\left(M_{\mathscr{P}}(t)-M_{\mathscr{P} l}(t)\right)(u \otimes \psi(f)),\left(M_{\mathscr{P}}(t)-M_{\mathscr{P} \prime}(t)\right)(u \otimes \psi(f))\right\rangle \\
=\int_{0}^{t}\left\{2 \operatorname { R e } \left\langle\left(\left(M_{\mathscr{P}}(s)-M_{\mathfrak{P}^{\prime}}(s)\right)(u \otimes \psi(f)),\right.\right.\right. \\
\left.\quad \sum_{j=0}^{n} \sum_{l=0}^{n_{l}} K\left(f, f, P\left(\Delta_{j_{l}}\right) x, P\left(\Delta_{J_{l}}\right), P\left(\Delta_{j_{l}}\right) y ; s\right)(u \otimes \psi(f))\right\rangle \\
\left.\quad+\sum_{J=0}^{n} \sum_{l=0}^{n_{J}}\left\|L\left(f, P\left(\Delta_{j_{l}}\right) x, P\left(\Delta_{j_{l}}\right) ; s\right)(u \otimes \psi(f))\right\| \|^{2}\right\} d s
\end{gathered}
$$




$$
\begin{aligned}
& \leqq \int_{0}^{t}\left\{\left\|\left(M_{\mathscr{P}}(s)-M_{\mathscr{P} \prime}(s)\right)(u \otimes \psi(f))\right\|^{2}\right. \\
& +\left(\sum_{j=0}^{n} \sum_{l=0}^{n_{j}}\left\|K\left(f, f, P\left(\Delta_{J_{l}}\right) x, P\left(\Delta_{j_{l}}\right), P\left(\Delta_{j_{l}}\right) y ; s\right)(u \otimes \psi(f))\right\|\right)^{2} \\
& \left.+\sum_{j=0}^{n} \sum_{l=0}^{n_{j}}\|\| L\left(f, P\left(\Delta_{J l}\right) x, P\left(\Delta_{j_{l}}\right) ; s\right)(u \otimes \psi(f)) \|^{2}\right\} d s .
\end{aligned}
$$

Now use the estimates (2.8) and (2.10) and the integrating factor $e^{-t}$ to obtain the required result with

$$
C(T)=e^{T / 2} \sup _{0 \leqq s \leqq T} \max \left\{2 \sqrt{3} \beta(x, y, f, I ; s),(2 \gamma(x, f, I ; s))^{1 / 2}\right\} .
$$

Now let $\left(\mathscr{P}_{n}, n \in \mathbb{N}\right)$ be a sequence of partitions of $[a, b]$ with $\lim _{n \rightarrow \infty}\left|\mathscr{P}_{n}\right|=0$ and write $M_{n}(t)=M_{\dddot{p}_{n}}(t)$ for each $n \in \mathbb{N}, t \in \mathbb{R}^{+}$.

Lemma 3.2. For each $u \in \mathfrak{D}_{0}, f \in \mathscr{S}, T \in \mathbb{R}^{+}$, the sequence $\left(M_{n}(t)(u \otimes \psi(f))\right)_{n \in \mathbb{N}}$ converges in $\mathfrak{H}$, uniformly for $0 \leqq t \leqq T$.

Proof. Let $m, n \in \mathbb{N}$, then by Lemma 3.1 we have

$$
\begin{aligned}
& \left\|M_{n}(t)(u \otimes \psi(f))-M_{m}(t)(u \otimes \psi(f))\right\| \\
& \quad=\left\|\left(M_{n}(t)-M_{m}(t)\right)(u \otimes \psi(f))\right\| \\
& \quad \leqq\left\|\left(M_{n}(t)-M_{\mathscr{P}_{n} V \mathscr{P}_{m}}(t)\right)(u \otimes \psi(f))\right\|+\left\|\left(M_{m}(t)-M_{\mathscr{P}_{n} V \mathscr{P}_{m}}(t)\right)(u \otimes \psi(f))\right\| \\
& \quad \leqq C_{T}\left(\gamma_{T}\left(u, f ; \mathscr{P}_{n}\right)+\gamma_{T}\left(u, f ; \mathscr{P}_{m}\right)\right)
\end{aligned}
$$

$\rightarrow 0$ as $n, m \rightarrow \infty$ by the admissibility condition.

So the sequence is uniformly Cauchy and hence uniformly convergent in $\mathfrak{H}$.

For each $u \in \mathfrak{D}_{0}, f \in S, t \in \mathbb{R}^{+}$, we write

$$
M_{a, b}(t)(u \otimes \psi(f))=\lim _{n \rightarrow \infty} M_{n}(t)(u \otimes \psi(f)) .
$$

Clearly each such $M_{a, b}(t)$ is a well-defined linear operator on $\mathfrak{H}$ with $\mathfrak{D}_{0} \otimes \mathscr{E}(\mathscr{S}) \subseteq$ $\operatorname{Dom}\left(M_{a, b}(t)\right)$ and the process $M_{a, b}=\left(M_{a, b}(t), t \in \mathbb{R}^{+}\right)$is adapted.

We denote each $M_{a, b}(t)$ by

$$
\begin{gathered}
M_{a, b}(t)=\int_{a}^{b} \int_{0}^{t}\left\{E_{1}(s, \lambda) A_{x}^{\dagger}(d s, P(d \lambda))+E_{2}(s, \lambda) \Lambda(d s, P(d \lambda))\right. \\
\left.+E_{3}(s, \lambda) A_{y}(d s, P(d \lambda))\right\}
\end{gathered}
$$

and note that the integral is linear in each of the three arguments $E_{j}(j=1,2,3)$.

We now introduce some families of Borel measures which we will employ in the sequel. Fix $x, y \in \mathfrak{H}_{1}$ and with regard to the notation used in (2.5) and (2.6) define for each $f, g \in \mathscr{S}, t \in \mathbb{R}^{+}, F \in \mathscr{B}(\mathbb{R})$,

$$
K(F)(f, g ; t)=K(f, g, P(F) x, P(F), P(F) y ; t)
$$


(wherein $E_{4}$ is taken to be identically zero and to save the notation becoming too clumsy, we have suppressed the $\lambda$-dependence of $K$ through the operators $E_{J}(t, \lambda)$ ).

Let $A$ be any adapted process in $\mathfrak{H}$ and let $u, v \in \mathfrak{H}_{0}$, then $\int_{F}\langle A(t)(u \otimes$ $\psi(f)), K(d \lambda)(f, g ; t)(v \otimes \psi(g))\rangle$ is a finite Borel measure on $\mathbb{R}$.

Define a further family $\beta(\cdot)(f ; t)$ of finite Borel measures on $\mathbb{R}$ by

$$
\beta(F)(f ; t)=\beta(x, y, f, P(F) ; t),
$$

where $\beta$ is defined by (2.9) and observe that by (2.8) we have

$$
\|K(F)(f, f, t)\| \leqq 2(3 \phi(s))^{1 / 2} \beta(F)(f, t) .
$$

Similarly if we define for each $h \in \mathscr{S}, j=1,2$,

$$
L^{j}(F)(h ; t)=L^{j}(h, P(F) x, P(F) ; t),
$$

then $\left\langle\left\langle L^{1}(\cdot)(f ; t)(u \otimes \psi(f)), L^{2}(\cdot)(g ; t)(v \otimes \psi(g))\right\rangle\right\rangle$ is a finite Borel measure on $\mathbb{R}$ and defining the finite Borel measures $\gamma(\cdot)(f, t)$ by

$$
\gamma(F)(f ; t)=\gamma(x, f, P(F) ; t),
$$

where $\gamma$ is defined by $(2.11)$ we find by $(2.10)$ that

$$
\text { II } L(F)(f, t)(u \otimes \psi(f))\|\|^{2} \leqq 2 \phi(t) \gamma(F)(f, t) .
$$

Finally we define a family $\tau(\cdot)(f ; t)$ of finite Borel measures on $\mathbb{R}$ by

$$
\tau(\cdot)(f ; t)=12 \beta(\mathbb{R})(f ; t) \beta(\cdot)(f ; t)+2 \gamma(\cdot)(f ; t) .
$$

Lemma 3.3. For each $\phi \in \mathfrak{H}, u \in \mathfrak{D}_{0}, f, g, h \in \mathscr{S}, t \in \mathbb{R}^{+}$we have

$$
\int_{0}^{t} \int_{a}^{b}\langle\phi, E(s, \lambda)(u \otimes \psi(f))\rangle(g(s), P(d \lambda) h(s)) d s<\infty
$$

whenever $E$ is admissible on $[a, b]$.

Proof. Using the Schwarz inequality and the admissibility criterion we find

$$
\begin{aligned}
& \int_{0}^{t} \int_{a}^{b}\langle\phi, E(s, \lambda)(u \otimes \psi(f))\rangle\|P(d \lambda) h(s)\|_{\mathfrak{F}_{1}}^{2} d s \\
& \quad \leqq t\|\phi\| \sup _{a \leqq i \leqq b} \sup _{0 \leqq s \leqq t}\|E(s, \lambda)(u \otimes \psi(f))\| \sup _{0 \leqq s \leqq t}\|h(s)\|_{\mathfrak{F}_{1}}^{2} .
\end{aligned}
$$

Hence, by the polarisation identity, the integral in the statement of the lemma is also finite.

Theorem 3.4. For each $t \in \mathbb{R}^{+}, u, v \in \mathfrak{D}_{0}, f, g \in \mathscr{S}$ we have

(i) $\begin{aligned}\left\langle u \otimes \psi(f), M_{a, b}(t)(v \otimes \psi(g))\right\rangle= & \int_{0}^{t} \int_{a}^{b}\langle u \otimes \psi(f), K(d \lambda)(f, g ; s) \\ & \times(v \otimes \psi(g))\rangle d s .\end{aligned}$

$$
\times(v \otimes \psi(g))\rangle d s .
$$


(ii) If $M_{a, b}^{j}$ are processes of the form (3.1) with respect to the admissible triples $E_{k}^{j}(j=1,2, k=1,2,3$ respectively) then

$$
\begin{aligned}
& \left\langle M_{a, b}^{1}(t)(u \otimes \psi(f)), M_{a, b}^{2}(t)(v \otimes \psi(g))\right\rangle \\
& =\int_{0}^{t} \int_{a}^{b}\left\{\left\langle M_{a, b}^{1}(s)(u \otimes \psi(f)), K^{2}(d \lambda)(f, g ; s)(v \otimes \psi(g))\right\rangle\right. \\
& \quad+\left\langle K^{1}(d \lambda)(g, f ; s)(u \otimes \psi(f)), M_{a, b}^{2}(s)(v \otimes \psi(g))\right\rangle \\
& \left.\quad+\left\langle\left\langle L^{1}(d \lambda)(f ; s)(u \otimes \psi(f)), L^{2}(d \lambda)(g ; s)(v \otimes \psi(g))\right\rangle\right\rangle\right\} d s .
\end{aligned}
$$

(iii) For all $0 \leqq t \leqq T, T \in \mathbb{R}^{+}$,

$$
\left\|M_{a, b}(t)(u \otimes \psi(f))\right\|^{2} \leqq \int_{0}^{T} \int_{a}^{b} e^{t-s} \phi(s, \lambda) \tau(d \lambda)(f ; s) d s,
$$

where

$$
\phi(s, \lambda)=\sum_{j=1}^{3}\left\|E_{j}(s, \lambda)(u \otimes \psi(f))\right\|^{2}
$$

\section{Proof.}

(i) For simplicity we take $E_{2}$ and $E_{3}$ to be identically zero.

Note that by Lemma 3.3, the right-hand side of (3.2) is finite. Using Theorem 3.2 we find

$$
\begin{aligned}
\langle u & \left.\otimes \psi(f), M_{a, b}(t)(v \otimes \psi(g))\right\rangle=\lim _{n \rightarrow \infty}\left\langle u \otimes \psi(f), M_{n}(t)(v \otimes \psi(g))\right\rangle \\
& =\lim _{n \rightarrow \infty} \int_{0}^{t} \sum_{j=0}^{m_{n}}\left\langle u \otimes \psi(f),\left(f(s), P\left(\lambda_{j}^{n}, \lambda_{j+1}^{n}\right) x\right) E_{1}\left(s, \mu_{j}^{n}\right)(v \otimes \psi(g))\right\rangle,
\end{aligned}
$$

where $M_{n}(t)=M_{P_{n}}(t), P_{n}$ is the partition $\left\{a=\lambda_{0}^{n}<\lambda_{1}^{n}<\ldots<\lambda_{m_{n}+1}^{n}=b\right\}$ and each $\lambda_{j}^{n} \leqq \mu_{j}^{n} \leqq \lambda_{j+1}^{n}\left(0 \leqq j \leqq m_{n}\right)$.

We then find that

$$
\begin{gathered}
\lim _{n \rightarrow \infty}\left|\left\langle u \otimes \psi(f), M_{n}(t)(v \otimes \psi(g))\right\rangle-\int_{0}^{t} \int_{a}^{b}\langle u \otimes \psi(f), K(d \lambda)(f, g ; s)(v \otimes \psi(g))\rangle d s\right| \\
\leqq \lim _{n \rightarrow \infty} \int_{0}^{t} \mid\left\langle u \otimes \psi(f),\left\{\sum_{j=0}^{m_{n}}\left(f(s), P\left(\lambda_{j}^{n}, \lambda_{j+1}^{n}\right) x\right) E_{1}\left(s, \mu_{j}\right)(v \otimes \psi(g))\right.\right. \\
\left.\left.\quad-\int_{a}^{b}(f(s), P(d \lambda) x) E_{1}(s, \lambda)(v \otimes \psi(g))\right\rangle\right\} \mid d s
\end{gathered}
$$

$=0$, where the interchange of limit and integral is justified by the dominated convergence theorem.

(ii) is proved similarly. 
(iii) Arguing as in the proof of Lemma 3.1 and using (3.7) yields

$$
\begin{aligned}
& \left\|M_{a, b}(t)(u \otimes \psi(f))\right\|^{2} \\
& \begin{array}{l}
\int_{0}^{t}\left\{2 \operatorname{Re}\left\langle M_{a, b}(s)(u \otimes \psi(f)), \int_{a}^{b} K(d \lambda)(f, f ; s)(u \otimes \psi(f))\right\rangle\right. \\
\left.\quad+\int_{0}^{t} \int_{a}^{b}\|L(d \lambda)(f ; s)(u \otimes \psi(f))\| \|^{2}\right\} d s
\end{array} \\
& \leqq \int_{0}^{t}\left\{\left\|M_{a, b}(s)(u \otimes \psi(f))\right\|^{2}+\left(\int_{a}^{b}\|K(d \lambda)(f, f ; s)(u \otimes \psi(f))\|\right)^{2}\right. \\
& \left.\quad+\left.\int_{0}^{t} \int_{a}^{b}\|L(d \lambda)(f ; s)(u \otimes \psi(f))\|\right|^{2}\right\} d s
\end{aligned}
$$

The required result now follows on using the estimates (3.3)-(3.4), the definition (3.5) and the fact that if $m$ is a finite measure on $[a, b]$ and $f \in L^{2}([a, b], m)$, then

$$
\left(\int_{a}^{b} f(\lambda) m(d \lambda)\right)^{2} \leqq m(a, b) \int_{a}^{b}|f(\lambda)|^{2} m(d \lambda)
$$

Using (3.8) and a similar argument to that in [HuPa 1], it is easily deduced that for each $u \in \mathfrak{D}_{0}, f \in \mathscr{S}, T \in \mathbb{R}$, the map $t \rightarrow M_{a, b}(t)(u \otimes \psi(f))$ is continuous from $[0, T]$ into $\mathfrak{H}$.

Let $E=\left(E(t, \lambda), t \in \mathbb{R}^{+}, \lambda \in \mathbb{R}\right)$ be a family in $\mathfrak{H}$. We say that it is strongly admissible if

(i) Each $E(\lambda) \in L_{\text {loc }}^{2}\left(\mathfrak{D}_{0}, \mathscr{S}\right)$.

(ii) $E$ is $\lambda$-continuous on the whole of $\mathbb{R}$.

(iii) For each $u \in \mathfrak{D}_{0}, f \in \mathscr{S}, t \in \mathbb{R}^{+}$the map

$\lambda \rightarrow \sup _{0 \leqq s \leqq t}\|E(s, \lambda)(u \otimes \psi(f))\|$ from $\mathbb{R}$ to $\mathbb{R}^{+}$is bounded.

Lemma 3.5. Let $M_{a, b}$ be the stochastic integral of a strongly admissible triple, then for each $u \in \mathfrak{D}_{0}, f \in \mathscr{S}, t \in \mathbb{R}^{+}$,

$$
\lim _{a \rightarrow-\infty} \lim _{b \rightarrow \infty} M_{a, b}(t)(u \otimes \psi(f)) \quad \text { exists in } \mathfrak{H} .
$$

Proof. Let $c>b>a$, then it is easy to see that

$$
M_{a, c}(t)=M_{a, b}(t)+M_{b, c}(t) .
$$

Hence by (3.8), we have for each $T \in \mathbb{R}^{+}, 0 \leqq t \leqq T$,

$$
\begin{aligned}
& \left\|M_{a, c}(t)(u \otimes \psi(f))-M_{a, b}(t)(u \otimes \psi(f))\right\|^{2} \\
& =\left\|M_{b, c}(t)(u \otimes \psi(f))\right\|^{2} \\
& \leqq \int_{0}^{T} \int_{b}^{c} e^{t-s} \phi(s, \lambda) \tau(d \lambda)(f ; s) d s \\
& \leqq e^{T} \int_{b}^{c} \sup _{0 \leqq s \leqq T} \phi(s, \lambda) \sigma(d \lambda)(f ; T)
\end{aligned}
$$


$\rightarrow 0$ as $b, c \rightarrow \infty$, where $\sigma().(f, T)=\int_{0}^{T} \tau().(f ; s) d s$ is a finite Borel measure on $\mathbb{R}$. Hence $\lim _{b \rightarrow \infty} M_{a, b}(t)(u \otimes \psi(f))$ exists.

The other limit is obtained similarly.

We write $M(t)(u \otimes \psi(f))=\lim _{a \rightarrow-\infty} \lim _{b \rightarrow \infty} M_{a, b}(t)(u \otimes \psi(f))$, and denote the adapted process $M=\left(M(t), t \in \mathbb{R}^{+}\right)$as in (3.1). In the following we will include a "drift" term and write

$$
\begin{gathered}
M(t)=\int_{-\infty}^{\infty} \int_{0}^{t}\left\{E_{1}(s, \lambda) A_{x}^{\dagger}(d s, P(d \lambda))+E_{2}(s, \lambda) \Lambda(d s, P(d \lambda))\right. \\
\left.+E_{3}(s, \lambda) A_{y}(d s, P(d \lambda))\right\}+\int_{0}^{t} E_{4}(s) d s
\end{gathered}
$$

where $E_{4} \in L_{\text {loc }}^{2}\left(\mathfrak{D}_{0}, \mathscr{S}\right)$.

Arguing as in [HuPa 1], we obtain the following generalised quantum Ito product formula from theorem 3.4 (ii).

Theorem 3.6. Let $M^{j}$ be processes of the form (3.6) with respect to the strongly admissible triples $E_{k}^{j}\left(j=1,2, k=1,2,3\right.$ respectively) such that each $M^{J}(t), E_{k}^{j}$ $(t, \lambda) \in B(\mathfrak{S})$, for $t \in \mathbb{R}^{+}, \lambda \in \mathbb{R}$ and

$$
\sup _{\lambda \in \mathbb{R}} \max \sup _{0 \leqq s \leqq t}\left\{\left\|M^{j}(s)\right\|,\left\|E_{k}^{j}(s, \lambda)\right\|,\left\|E_{4}(s)\right\|, j=1,2, k=1,2,3,\right\}<\infty,
$$

$M^{1} M^{2}=\left(M^{1}(t) M^{2}(t), t \in \mathbb{R}^{+}\right)$is then a process of quantum spectral stochastic integrals with

$$
\begin{aligned}
M^{1}(t) M^{2}(t)=\int_{-\infty}^{\infty} \int_{0}^{t}\{ & {\left[M^{1}(s) E_{1}^{2}(s, \lambda)+E_{1}^{1}(s, \lambda) M^{2}(s)+E_{2}^{1}(s, \lambda) E_{1}^{2}(s, \lambda)\right] } \\
& \times A_{x}^{\dagger}(d s, P(d \lambda))+\left[M^{1}(s) E_{2}^{2}(s, \lambda)+E_{2}^{1}(s, \lambda) M^{2}(s)\right. \\
& \left.+E_{2}^{1}(s, \lambda) E_{2}^{2}(s, \lambda)\right] \Lambda(d s, P(d \lambda))+\left[M^{1}(s) E_{3}^{2}(s, \lambda)\right. \\
& \left.\left.+E_{3}^{1}(s, \lambda) M^{2}(s)+E_{3}^{1}(s, \lambda) E_{2}^{2}(s, \lambda)\right] A_{y}(d s, P(d \lambda))\right\} \\
& +\int_{0}^{t}\left\{M^{1}(s) E_{4}^{2}(s)+E_{4}^{1}(s) M^{2}(s)\right. \\
& \left.+\int_{-\infty}^{\infty} E_{3}^{1}(s, \lambda) E_{1}^{2}(s, \lambda)(y, P(d \lambda) x)\right\} d s
\end{aligned}
$$

Notes. (i) The interchange of integrals in the final term of (3.10) is justified by Fubini's theorem and $\int_{-\infty}^{\infty} E_{3}^{1}(s, \lambda) E_{1}^{2}(s, \lambda)(y, P(d \lambda) x)$ is a standard $B(\mathfrak{h})$-valued integral.

(ii) It is tempting to introduce the simplified notation for (3.9) of

$$
\begin{gathered}
d M(t)=\int_{-\infty}^{\infty}\left\{E_{1}(t, \lambda) A_{x}^{\dagger}(d t, P(d \lambda))+E_{2}(t, \lambda) \Lambda(d t, P(d \lambda))\right. \\
\left.+E_{3}(t, \lambda) A_{y}(d t, P(d \lambda))\right\}+E_{4}(t) d t
\end{gathered}
$$

Using this notation we can write (3.10) in the following simpler form

$$
d\left(M^{1}(t) M^{2}(t)\right)=d M^{1}(t) \cdot M^{2}(t)+M^{1}(t) \cdot d M^{2}(t)+d M^{1}(t) \cdot d M^{2}(t),
$$


where the term $d M^{1}(t) \cdot d M^{2}(t)$ is evaluated by bilinear extension of the rule that all products of differentials vanish with the exception of

$$
\begin{gathered}
A_{y}\left(d t, P\left(F_{1}\right)\right) \cdot A_{x}^{\dagger}\left(d t, P\left(F_{2}\right)\right)=\left(y, P\left(F_{1} \cap F_{2}\right) x\right) d t, \\
A_{y}\left(d t, P\left(F_{1}\right)\right) \cdot \Lambda\left(d t, P\left(F_{2}\right)\right)=A_{y}\left(d t, P\left(F_{1} \cap F_{2}\right)\right), \\
\Lambda\left(d t, P\left(F_{1}\right)\right) \cdot A_{x}^{\dagger}\left(d t, P\left(F_{2}\right)\right)=A_{x}^{\dagger}\left(d t, P\left(F_{1} \cap F_{2}\right)\right), \\
\Lambda\left(d t, P\left(F_{1}\right)\right) \cdot \Lambda\left(d t, P\left(F_{2}\right)\right)=\Lambda\left(d t, P\left(F_{1} \cap F_{2}\right)\right),
\end{gathered}
$$

where $F_{1}, F_{2} \in \mathscr{B}(\mathbb{R})$.

Despite its simplicity, we will not use this notation in the sequel as it misleadingly suggests that the time integral precedes the spectral one. Although we obtain some results justifying such an interchange in the next section, it is not guaranteed that the required conditions hold for the processes we will consider in the final two sections.

We close this section with a result on the "linear independence" of stochastic differentials which we will find useful in Sect. 5 below. Let $M$ be a process of the type described in Theorem 3.6 satisfying the additional condition that each of the maps

$$
t \rightarrow E_{j}(t, \lambda)(u \otimes \psi(f)), \quad t \rightarrow E_{4}(t)(u \otimes \psi(f))
$$

is continuous from $\mathbb{R}^{+}$into $\mathfrak{H}$ for $j=1,2,3$ and each $u \in \mathfrak{D}_{0}, f \in \mathscr{S}, \lambda \in \mathbb{R}$.

I am grateful to Martin Brooks for providing the proof of the following

Theorem 3.7. If $M(t)=0$ for all $t \in \mathbb{R}^{+}$on $\mathfrak{D}_{0} \underline{\otimes} \mathscr{E}(\mathscr{S})$, then

$$
E_{j}(t, \lambda)=E_{4}(t)=0 \text { on } \mathfrak{D}_{0} \underline{\otimes} \mathscr{E}(\mathscr{S})
$$

for each $j=1,2,3, \lambda \in \mathbb{R}, t \in \mathbb{R}^{+}$.

Proof. Suppose that $M(t)=0$ for some $t \in \mathbb{R}^{+}$, then differentiating (3.6) yields

$$
\int_{-\infty}^{\infty}\langle u \otimes \psi(f), K(d \lambda)(f, g ; t)(v \otimes \psi(g))\rangle+\left\langle u \otimes \psi(f), E_{4}(t)((v \otimes \psi(g))\rangle=0 .\right.
$$

Choosing $f$ and $g$ to be continuous and such that $f(t)=g(t)=0$ yields $E_{4}(t)((v \otimes$ $\psi(g))=0$ as in [Par] Proposition 27.3, p.224.

Now let $f$ be continuous and such that $f(t)=0$. By (3.7) we find that

$$
\begin{aligned}
0= & \frac{d}{d t}\|M(t)(u \otimes \psi(f))\|^{2} \\
= & \int_{-\infty}^{\infty}\left\|E_{1}(t, \lambda)(u \otimes \psi(f))\right\|^{2}\|P(d \lambda) x\|^{2} \\
& +\int_{-\infty}^{\infty} 2 \operatorname{Re}\left\langle E_{1}(t, \lambda)(u \otimes \psi(f)), E_{2}(t, \lambda)(u \otimes \psi(f))\right\rangle(P(d \lambda) x, f(t)) \\
& +\int_{-\infty}^{\infty}\left\|E_{2}(t, \lambda)(u \otimes \psi(f))\right\|^{2}\|P(d \lambda) f(t)\|^{2} .
\end{aligned}
$$


Hence each $E_{1}(t, \lambda)=0$. Upon relaxing the restriction $f(t)=0$, we see that each $E_{2}(t, \lambda)=0$.

Now a standard argument shows that each $M(t)$ is closeable and the restriction of its adjoint to $\mathfrak{D}_{0} \underline{\otimes} \mathscr{E}(\mathscr{S})$ is

$$
\left.M(t)^{\dagger}=\int_{0}^{t} \int_{-\infty}^{\infty} E_{3}(s, \lambda)^{*} A^{\dagger}(d s, P(d \lambda) x)\right)
$$

A similar argument to the above then shows that each $E_{3}(s, \lambda)=0$

\section{Interchange of Integrals}

In the last section we constructed, as an adapted process in $\mathfrak{H}$, integrals of the form

$$
\begin{gathered}
M_{a, b}(t)=\int_{a}^{b} \int_{0}^{t}\left\{E_{1}(s, \lambda) A_{x}^{\dagger}(d s, P(d \lambda))+E_{2}(s, \lambda) \Lambda(d s, P(d \lambda)\right. \\
\left.+E_{3}(s, \lambda) A_{y}(d s, P(d \lambda))\right\}
\end{gathered}
$$

for the admissible families $E_{j}(j=1,2,3)$.

In this section we will demonstrate that it makes sense to interchange the integrals in (4.1). We begin by assuming that for each $\lambda \in[a, b], j=1,2,3, E_{j}(\lambda)=$ $\left(E_{j}(t, \lambda), t \in \mathbb{R}^{+}\right) \in \Sigma\left(\mathfrak{D}_{0}, \mathscr{S}\right)$ with respect to the common partition $\left\{0=t_{0}<t_{1}<\right.$ $\left.\cdots<t_{n} \rightarrow \infty\right\}$. In this case we can clearly interchange the integrals in (4.1) to write

$$
\begin{aligned}
M_{a, b}(t)=\sum_{n=0 a}^{\infty} \int_{a}^{b} & E_{1}\left(t_{n} \wedge t, \mu\right) a^{\dagger}\left(\chi_{\left[t_{n} \wedge t, t_{n+1} \wedge t\right)} \otimes P(d \mu) x\right) \\
& +E_{2}\left(t_{n} \wedge t, \mu\right) \lambda\left(\chi_{\left[t_{n} \wedge t,_{n+1} \wedge t\right)} \otimes P(d \mu)\right) \\
& \left.+E_{3}\left(t_{n} \wedge t, \mu\right) a\left(\chi_{\left[t_{n} \wedge t, t_{n+1} \wedge t\right)} \otimes P(d \mu) y\right)\right\} .
\end{aligned}
$$

Now for $j=1,2,3$ let $\left(E_{j}^{(n)}, n \in \mathbb{N}\right)$ be sequences of admissible families wherein each $E_{j}^{(n)}(\mu) \in \Sigma\left(\mathfrak{D}_{0}, \mathscr{S}\right)$ and converges to $E_{j}(\mu) \in L_{\text {loc }}^{2}\left(\mathfrak{D}_{0}, \mathscr{S}\right)$ in the sense of (2.1), where each $E_{j}$ is admissible. Examples of such sequences are not difficult to manufacture, e.g. take each $E_{j}^{(n)}(t, \mu)=F_{j}^{(n)}(t) f_{j}(\mu)$, where $f_{j} \in C(a, b)$ and $F_{j}^{(n)} \in$ $\Sigma\left(\mathfrak{D}_{0}, \mathscr{S}\right)$ with $\sup _{n \in \mathbb{N}} \sup _{0 \leqq s \leqq t}\left\|F_{j}^{(n)}(s)(u \otimes \psi(f))\right\|<\infty$ and $\lim _{n \rightarrow \infty} \sup _{0 \leqq s \leqq t} \|$ $\left(F_{j}^{(n)}(s)-F_{J}(s)\right)(u \otimes \psi(f)) \|=0$.

We define a sequence of stochastic integrals $\left(M_{a, b}^{(n)}, n \in \mathbb{N}\right)$, where each $M_{a, b}^{(n)}$ is defined by (4.2) wherein each $E_{J}$ on the right-hand side is replaced by $E_{j}^{(n)}$, where $j=1,2,3$ respectively. We then obtain the following

Lemma 4.1. For each $u \in \mathfrak{D}_{0}, f \in \mathscr{S}, t \in \mathbb{R}^{+},-\infty<a<b<\infty$,

$$
\lim _{n \rightarrow \infty} M_{a, b}^{(n)}(t)(u \otimes \psi(f)) \quad \text { exists in } \mathfrak{H} \text {. }
$$


Proof. Using (3.8) and the admissibility condition we find that $\left\|\left(M_{a, b}^{(n)}(t)(u \otimes \psi(f))-M_{a, b}^{(m)}(t)(u \otimes \psi(f))\right)\right\|^{2} \leqq \int_{0}^{T} \int_{a}^{b} e^{t-s} \phi^{(n, m)}(s, \lambda) \tau(d \lambda)(f ; s) d s$, where

Hence

$$
\phi^{(n, m)}(s, \lambda)=\sum_{j=1}^{3}\left\|\left(E_{j}^{(n)}(s, \lambda)-E_{j}^{(m)}(s, \lambda)\right)(u \otimes \psi(f))\right\|^{2} .
$$

$$
\begin{aligned}
& \left\|\left(M_{a, b}^{(n)}(t)(u \otimes \psi(f))-M_{a, b}^{(m)}(t)(u \otimes \psi(f))\right)\right\|^{2} \\
& \quad \leqq \int_{0}^{T} e^{t-s} \sup _{a \leqq i \leqq b} \phi^{(n, m)}(s, \lambda) \tau(I)(f, s) d s \\
& \quad \rightarrow \text { aas } n, m \rightarrow \infty \text { by }(2.1) .
\end{aligned}
$$

The required result then follows.

We write $\hat{M}_{a, b}(t)(u \otimes \psi(f))=\lim _{n \rightarrow \infty} M_{a, b}^{(n)}(t)(u \otimes \psi(f))$. Clearly each $\hat{M}_{a, b}(t)$ is a linear operator in $\mathfrak{H}$ and the process $\left(\hat{M}_{a, b}(t), t \in \mathbb{R}^{+}\right)$is adapted. We write for each $t \in \mathbb{R}^{+}$,

$$
\begin{aligned}
\hat{M}_{a, b}(t)=\int_{0}^{t} \int_{a}^{b}\{ & E_{1}(s, \hat{\lambda}) A_{x}^{\dagger}(d s, P(d \lambda)) \\
& \left.+E_{2}(s, \lambda) \Lambda(d s, P(d \lambda))+E_{3}(s, \lambda) A_{y}(d s, P(d \lambda))\right\} .
\end{aligned}
$$

Theorem 4.2. For each $u, v \in \mathfrak{D}_{0}, f, g \in \mathscr{S}, t \in \mathbb{R}^{+}$, we have

$$
\left\langle u \otimes \psi(f), \hat{M}_{a, b}(t)(v \otimes \psi(g))\right\rangle=\int_{0}^{t} \int_{a}^{b}\langle u \otimes \psi(f), K(d \lambda)(f, g ; s)(v \otimes \psi(g))\rangle d s .
$$

Proof. As in the proof of Theorem 3.4 (i), we take $E_{2}=E_{3}=0$ and observe that, by (3.6),

$$
\begin{aligned}
\langle u & \left.\otimes \psi(f), \hat{M}_{a, b}(t)(v \otimes \psi(g))\right\rangle \\
& =\lim _{n \rightarrow \infty}\left\langle u \otimes \psi(f), M_{a, b}^{(n)}(t)(v \otimes \psi(g))\right\rangle \\
& =\lim _{n \rightarrow \infty} \int_{0}^{t} \int_{a}^{b}\left\langle u \otimes \psi(f), E_{1}^{(n)}(s, \lambda)(v \otimes \psi(g))\right\rangle(f(s), P(d \lambda) x) d s .
\end{aligned}
$$

Now several applications of the Schwarz inequality yield

$$
\begin{aligned}
\mid\langle u & \otimes \psi(f), M_{a, b}^{(n)}(t)(v \otimes \psi(g))-\int_{0}^{t} \int_{a}^{b}\langle u \otimes \psi(f), K(d \lambda)(f, g ; s)(v \otimes \psi(g))\rangle d s \mid \\
& =\mid \int_{0}^{t} \int_{a}^{b}\left\langle u \otimes \psi(f),\left(E_{1}^{(n)}(s, \lambda)-E_{1}(s, \lambda)\right)((v \otimes \psi(g))\rangle(f(s), P(d \lambda) x) d s\right|
\end{aligned}
$$




$$
\begin{aligned}
\leqq & \int_{0}^{t} \sup _{a \leqq i \leqq b} \mid\left\langle u \otimes \psi(f),\left(E_{1}^{(n)}(s, \lambda)-E_{1}(s, \lambda)\right)((v \otimes \psi(g))\rangle \|(f(s), x)\right| d s \\
\leqq & \|u\| \cdot\|\psi(f)\| \cdot\|x\|_{\mathfrak{S}_{1}} \sup _{0 \leqq s \leqq t}\|f(s)\|_{\mathfrak{S}_{1}} \\
& \left.\times \int_{0}^{t} \sup _{a \leqq \lambda \leqq b} \| E_{1}^{(n)}(s, \lambda)-E_{1}(s, \lambda)\right)((v \otimes \psi(g)) \| d s \\
\leqq & C(t)\left(\int_{0}^{t} \sup _{a \leqq i \leqq b} \|\left(E_{1}^{(n)}(s, \lambda)-E_{1}(s, \lambda)\right)\left((v \otimes \psi(g)) \|^{2} d s\right)^{1 / 2}\right.
\end{aligned}
$$

$\rightarrow 0$ as $n \rightarrow \infty$ by the admissibility condition and (2.1), $C(t)$ in the final term being a positive constant.

Theorem 4.3. For each $-\infty<a<b<\infty, u \in \mathfrak{D}_{0}, f \in \mathscr{S}, t \in \mathbb{R}^{+}$, we have

$$
M_{a, b}(t)(u \otimes \psi(f))=\hat{M}_{a, b}(t)(u \otimes \psi(f)) .
$$

Proof. By Theorems 3.3(i) and 4.2 we have for each $u, v \in \mathfrak{D}_{0}, f, g \in \mathscr{S}$,

$$
\left\langle u \otimes \psi(f),\left(\hat{M}_{a, b}(t)-M_{a, b}(t)\right)(v \otimes \psi(g))\right\rangle=0,
$$

and the result follows from the fact that $\mathfrak{D}_{0} \otimes \mathscr{E}(\mathscr{S})$ is dense in $\mathfrak{H}$

These results are extended to the case $a=-\infty, b=\infty$ by taking limits as in Sect. 3 under the strong admissibility hypothesis.

\section{Quantum Stochastic Differential Equations and Dilations of Semigroups}

Let $\left\{L_{j}(\lambda), \lambda \in \mathbb{R}\right\}$ for $j=1,2,3$ be families of linear operators in $\mathfrak{H}_{0}$ and assume that for each $j=1,2,3$ we have

(i) $L_{j}(\lambda) \in B\left(\mathfrak{H}_{0}\right)$ for each $\lambda \in \mathbb{R}$.

(ii) The map $\lambda \rightarrow L_{j}(\lambda)$ is strongly continuous.

(iii) $\sup _{\lambda \in \mathbb{R}}\left\|L_{j}(\lambda)\right\|<\infty$.

Our first goal in this section is to investigate the existence of solutions to stochastic differential equations of the form

$$
\begin{gathered}
X(t)=I+\int_{-\infty}^{\infty} \int_{0}^{t}\left\{X(s) L_{1}(\lambda) A_{x}^{\dagger}(d s, P(d \lambda))+X(s) L_{2}(\lambda) \Lambda(d s, P(d \lambda))\right. \\
\left.+X(s) L_{3}(\lambda) A_{y}(d s, P(d \lambda))\right\}+\int_{0}^{t} X(s) L_{4} d t
\end{gathered}
$$

where $L_{4} \in B\left(\mathfrak{S}_{0}\right)$. Note that we will write all subsequent SDE's in integrated form as in (5.1) to avoid the notational difficulties discussed at the end of Sect. 3.

We take $\mathfrak{D}_{0}$ herein to be the whole of $\mathfrak{H}_{0}$. Where convenient we will use the notation $L_{4}(\lambda)=L_{4}$ for all $\lambda \in \mathbb{R}$.

Theorem 5.1. There exists a unique adapted process $X=\left(X(t), t \in \mathbb{R}^{+}\right)$satisfying (5.1) 
Proof. We first show that we can define a sequence of stochastic integrals inductively by

$$
\begin{gathered}
X_{0}(t)=I, \\
X_{n}(t)=I+\int_{-\infty}^{\infty} \int_{0}^{t}\left(X _ { n - 1 } ( s ) \left\{L_{1}(\lambda) A_{x}^{\dagger}(d s, P(d \lambda))+L_{2}(\lambda) \Lambda(d s, P(d \lambda))\right.\right. \\
\left.\left.+L_{3}(\lambda) A_{y}(d s, P(d \lambda))\right\}+X_{n-1}(s) L_{4} d s\right) .
\end{gathered}
$$

We must show that each of the families $X_{n}(t) L_{j}(\lambda)$ is strongly admissible. In fact we will prove a slightly stronger result namely that each $X_{n}(\cdot) L_{j}(\lambda) \in L_{\text {loc }}^{2}\left(\mathfrak{D}_{0}, \mathscr{S}\right)$, that the map $\lambda \rightarrow \sup _{0 \leqq s \leqq t}\left\|X_{n}(s) A L_{j}(\lambda)(u \otimes \psi(f))\right\|$ is bounded and that the family $X_{n}(t) A L_{j}(\lambda)$ is $\bar{\lambda}$-continuous on $\mathbb{R}$ for all $A \in B\left(\mathfrak{H}_{0}\right), u \in \mathfrak{D}_{0}, f \in \mathscr{S}$, $j=1,2,3,4$.

We demonstrate this by induction. It follows from conditions (i) to (iii) above that it holds for $n=1$. Suppose now that it holds for $n-1$. Square integrability is established by a similar argument to that of [HuPa 1], Proposition 7.1.

To show the continuity condition we use (3.8) to find that for each $T \in \mathbb{R}^{+}$, $j=1,2,3,4$,

$$
\begin{aligned}
& \sup _{0 \leqq t \leqq T}\left\|X_{n}(s) A\left(L_{j}(\lambda)-L_{j}(v)\right)(u \otimes \psi(f))\right\|^{2} \\
& \leqq \sup _{0 \leqq t \leqq T} \int_{0}^{t} \int_{-\infty}^{\infty} e^{t-s} \sum_{k=1}^{4}\left\|X_{n-1}(s) A\left(L_{j}(\lambda)-L_{j}(v)\right) L_{k}(\mu)(u \otimes \psi(f))\right\|^{2} \\
& \quad \times \tau(d \mu)(f, s) d s .
\end{aligned}
$$

By hypothesis each of the maps $\mu \rightarrow \sup _{0 \leqq t \leqq T} \| X_{n-1}(s) A\left(L_{J}(\lambda)-L_{j}(v)\right) L_{k}(\mu)(u \otimes$ $\psi(f)) \|$ is bounded and continuous. Hence we obtain,

$$
\begin{aligned}
& \sup _{0 \leqq t \leqq T}\left\|X_{n}(t) A\left(L_{j}(\lambda)-L_{J}(v)\right)(u \otimes \psi(f))\right\|^{2} \leqq e^{T} \int_{0}^{T} \tau(\mathbb{R})(f, s) d s . \\
& \times \sup _{\mu \in \mathbb{R}} \max _{1 \leqq J \leqq 4} \sup _{0 \leqq t \leqq T}\left\|X_{n-1}(t) A\left(L_{j}(\lambda)-L_{j}(v)\right)\left(\left(L_{k}(\mu) u\right) \otimes \psi(f)\right)\right\|^{2}
\end{aligned}
$$

from which the required result follows.

The boundedness condition is proved similarly.

To establish convergence of the sequence of iterates, we again argue as in [HuPa 1] above and using the estimate (3.8) we find that for $0 \leqq t \leqq T, u \in \mathfrak{H}_{0}$, $f \in \mathscr{S}$,

$$
\begin{aligned}
& \|\left(X_{n}(t)-X_{n-1}(t)(u \otimes \psi(f)) \|^{2}\right. \\
& \quad \leqq \frac{D(T)}{n !} \max _{1 \leqq j_{1}, \ldots, j_{n} \leqq 4} \sup _{\lambda \in \mathbb{R}}\left\|L_{j_{1}}(\lambda) \cdots L_{j_{n}}(\lambda) u\right\|^{2},
\end{aligned}
$$

where $D(T)>0$ is constant.

Hence for $n, m \in \mathbb{N}$,

$$
\begin{aligned}
& \sup _{0 \leqq t \leqq T} \|\left(\left(X_{n}(t)-X_{m}(t)(u \otimes \psi(f)) \|\right.\right. \\
& \quad \leqq \sum_{r=m+1}^{n}\left\{\max _{1 \leqq J_{1}, \ldots, J_{n} \leqq 4} \sup _{\lambda \in \mathbb{R}}\left\|L_{j_{1}}(\lambda)\right\| \cdots \sup _{\lambda \in \mathbb{R}}\left\|L_{j_{r}}(\lambda)\right\|\right\} \cdot\left(\frac{D(T)}{n !}\right)^{1 / 2}\|u\| \\
& \quad \rightarrow 0 \text { as } n, m \rightarrow \infty .
\end{aligned}
$$


Hence $\left(X_{n}(t)(u \otimes \psi(f))\right)_{n \in \mathbb{N}}$ converges uniformly in $\mathfrak{H}$ on $[0, T]$ and the solution to $(5.1)$ is given on $\mathfrak{H}_{0} \otimes \mathscr{E}(\mathscr{S})$ by

$$
X(t)(u \otimes \psi(f))=\lim _{n \rightarrow \infty} X_{n}(t)(u \otimes \psi(f)) .
$$

The $\lambda$-continuity of each of the families $X(t) L_{j}(\lambda)$ is established by an $\varepsilon / 3$ argument. The boundedness condition is easily established using the above estimates.

Uniqueness is proved by a similar argument to that of [ $\mathrm{HuPa} 1]$

In the sequel we will take $y=x$ in (5.1).

Theorem 5.2. A necessary and sufficient condition for the solution of (5.1) to consist of unitary operators in $\mathfrak{H}$ is that there exists a family $\{W(\lambda), \lambda \in \mathbb{R}\}$ of unitary operators on $\mathfrak{S}_{0}$ and a self-adjoint operator $H_{0} \in B\left(\mathfrak{S}_{0}\right)$ such that for each $\lambda \in \mathbb{R}$,

$$
\begin{aligned}
L_{2}(\lambda) & =W(\lambda)-I \\
L_{3}(\lambda) & =-L_{1}(\lambda)^{*} W(\lambda), \\
L_{4} & =i H_{0}-\frac{1}{2} \int_{-\infty}^{\infty} L_{1}(\lambda)^{*} L_{1}(\lambda)\|P(d \lambda) x\|^{2} .
\end{aligned}
$$

We omit the proof as it goes along the same lines as the corresponding result in [HuPa 1], making use of Theorem 3.6 and Theorem 3.7. We note that condition (iii) above guarantees the existence and boundedness of the $B\left(\mathfrak{S}_{0}\right)$-valued integral appearing in the expression for $L_{4}$.

From now on we will write $L$ in place of $L_{1}$ and denote the solution of (5.1) by $U=\left(U(t), t \in \mathbb{R}^{+}\right)$whenever it is unitary. Let $\mathscr{A}$ be a ${ }^{*}$-subalgebra of $B\left(\mathfrak{S}_{0}\right)$. (We can, of course, take $\mathscr{A}$ to be the whole of $B\left(\mathfrak{H}_{0}\right)$.)

We define a quantum stochastic flow $J=\left(j_{t}, t \in \mathbb{R}^{+}\right)$where each $j_{t}$ maps $\mathscr{A}$ into $B(\mathfrak{S})$ by the prescription

$$
j_{t}(a)=U(t) a U(t)^{*}
$$

for each $a \in \mathscr{A}, t \in \mathbb{R}^{+}$.

A standard calculation using Theorem 3.6 shows that each $j_{t}(a)$ satisfies the SDE

$$
\begin{gathered}
j_{t}(a)=a+\int_{-\infty}^{\infty} \int_{0}^{t}\left\{j_{s}\left(\alpha_{\lambda}(a)\right) A_{x}^{\dagger}(d s, P(d \lambda))+j_{s}\left(\beta_{\lambda}(a)\right) \Lambda(d s, P(d \lambda))\right. \\
\left.+j_{s}\left(\gamma_{\lambda}(a)\right) A_{x}(d s, P(d \lambda))\right\}+\int_{0}^{t} j_{s}(\delta(a)) d s
\end{gathered}
$$

where for each $\lambda \in \mathbb{R}$,

$$
\begin{aligned}
& \alpha_{\lambda}(a)=L(\lambda) a-W(\lambda) a W(\lambda)^{*} L(\lambda), \\
& \beta_{\lambda}(a)=W(\lambda) a W(\lambda)^{*}-a \\
& \gamma_{\lambda}(a)=a L(\lambda)^{*}-L(\lambda)^{*} W(\lambda) a W(\lambda)^{*}, \\
& \delta(a)=i\left[H_{0}, a\right] \\
&-\frac{1}{2} \int_{-\infty}^{\infty}\left\{L(\lambda)^{*} L(\lambda) a-2 L(\lambda)^{*} W(\lambda) a W(\lambda)^{*} L(\lambda)+a L(\lambda)^{*} L(\lambda)\right\}\|P(d \lambda) x\|^{2}
\end{aligned}
$$

(c.f. [Hud]). 
It is usual to assume that the operators $L(\hat{\lambda}), W(\lambda)$ and $H_{0}$ are such that each of the structure maps $\alpha_{\lambda}, \beta_{\lambda}, \gamma_{\lambda}$ and $\delta$ preserve the algebra $\mathscr{A}$.

Now take $\mathscr{A}=B\left(\mathfrak{H}_{0}\right)$, let $\mathbb{E}_{0}$ denote the vacuum conditional expectation on, $B(\mathfrak{H})$ and $T=\left(T_{t}, t \in \mathbb{R}^{+}\right)$be the norm continuous quantum dynamical semigroup on $B\left(\mathfrak{H}_{0}\right)$ whose infinitesimal generator is $\delta$, then we have a "stochastic dilation" of $T$ by

$$
T_{t}(a)=\mathbb{E}_{0}\left(j_{t}(a)\right)
$$

for each $t \in \mathbb{R}^{+}, a \in B\left(\mathfrak{H}_{0}\right)$.

We can construct a "coarser" dilation of $T$ as follows. It is a consequence of the work of Lindblad [Lin] that there exists a sequence $\left(M_{n}, n \in \mathbb{N}\right)$ in $B\left(\mathfrak{G}_{0}\right)$ with st- $\lim _{n \rightarrow \infty} \sum_{j=0}^{n} M_{n}^{*} M_{n}<\infty$ such that

$$
\delta(a)=i\left[H_{0}, a\right]+\frac{1}{2} \sum_{j=0}^{\infty}\left(M_{n}^{*} M_{n} a-2 M_{n}^{*} a M_{n}+a M_{n}^{*} M_{n}\right),
$$

so that $T$ can also be dilated using the technique of [HuPa 2]. We observe that there appears to be no direct construction of the $M_{n}$ 's from the $L(\lambda)$ 's.

We conclude this section by examining a class of mixed stochastic differential equations in which the formalism of [HuPa 1] is combined with ours. To this end let $x, y, w, z \in \mathfrak{H}_{1},\left(E_{j}, j=1,2,3\right)$ be strongly admissible families and $F_{j} \in L_{\text {loc }}^{2}\left(\mathfrak{D}_{0}, \mathscr{S}\right)$ for $j=1,2$. Consider the stochastic integral, $M=\left(M(t), t \in \mathbb{R}^{+}\right)$given by

$$
M(t)=M_{1}(t)+M_{2}(t),
$$

where

$$
\begin{gathered}
M_{1}(t)=\int_{0}^{t}\left(F_{1}(s) d A_{w}^{\dagger}(s)+F_{2}(s) d A_{z}(s)\right) \\
M_{2}(t)=\int_{-\infty}^{\infty} \int_{0}^{t}\left\{E_{1}(s, \lambda) A_{x}^{\dagger}(d s, P(d \lambda))+E_{2}(s, \lambda) \Lambda(d s, P(d \lambda))\right. \\
\left.\quad+E_{3}(s, \lambda) A_{y}(d s, P(d \lambda))\right\} .
\end{gathered}
$$

Recalling the notation of (2.7) and (3.8), we write for each $t \in \mathbb{R}^{+}, f \in \mathscr{S}$,

$$
\begin{gathered}
\zeta(t)=\max \left\{\phi(t), \sup _{i \in \mathbb{R}} \phi(t, \lambda)\right\}, \\
\rho(f, t)=2(\alpha(w, I, z ; t)+\tau(\mathbb{R})(f, t)) .
\end{gathered}
$$

We obtain the following

Lemma 5.3. For each $0 \leqq t \leqq T, u \in \mathfrak{D}_{0}, f \in \mathscr{S}$,

$$
\|M(t)(u \otimes \psi(f))\|^{2} \leqq \int_{0}^{T} e^{t-s} \zeta(s) \rho(f, s) d s .
$$


Proof. Using (2.4) and (3.8) we obtain

$$
\begin{aligned}
& \|M(t)(u \otimes \psi(f))\|^{2} \\
& \quad \leqq 2\left\|M_{1}(t)(u \otimes \psi(f))\right\|^{2}+2\left\|M_{2}(t)(u \otimes \psi(f))\right\|^{2} \\
& \quad \leqq 2 \int_{0}^{T} e^{t-s} \phi(s) \alpha(w, I, z ; s) d s+2 \int_{0}^{T} \int_{-\infty}^{\infty} e^{t-s} \phi(s, \lambda) \tau(d \lambda)(f ; s) d s \\
& \quad \leqq 2 \int_{0}^{T} e^{t-s} \phi(s) \alpha(w, I, z ; s) d s+2 \int_{0}^{T} e^{t-s} \sup _{\lambda \in \mathbb{R}} \phi(s, \lambda) \tau(\mathbb{R})(f ; s) d s \\
& \quad \leqq \int_{0}^{T} e^{t-s \zeta(s) \rho(f, s) d s \quad \text { as required . }}
\end{aligned}
$$

Let $L_{0} \in B\left(\mathfrak{H}_{0}\right)$ and $w \in \mathfrak{H}_{1}$ with $P(G) w=0$ for all $G \in \mathscr{B}(\mathbb{R})$. We take $L(\lambda), W(\lambda)$ (where $\lambda \in \mathbb{R}$ ) and $H_{0}$ to be as in the statement of Theorem 5.2. The following result is a straightforward extension of Theorems 5.1 and 5.2, where, in the existence proof, we use the estimate (5.5) in place of (3.8).

Theorem 5.4. There exists a unique unitary operator valued process $U=(U(t), t \in$ $\mathbb{R}^{+}$) which satisfies the $S D E$

$$
\begin{aligned}
U(t)= & I+\int_{0}^{t}\left(U(s) L_{0} d A_{w}^{\dagger}(s)-U(s) L_{0}^{*} d A_{w}(s)\right) \\
& +\int_{-\infty}^{\infty} \int_{0}^{t}\left\{U(s) L(\lambda) A_{x}^{\dagger}(d s, P(d \lambda))+U(s)(W(\lambda)-I) \Lambda(d s, P(d \lambda))\right. \\
& \left.\quad-U(s) L(\lambda)^{*} W(\lambda) A_{x}(d s, P(d \lambda))\right\} \\
& +\int_{0}^{t} U(s)\left\{i H_{0}-\frac{1}{2} L_{0}^{*} L_{0}\|w\|^{2}-\frac{1}{2} \int_{-\infty}^{\infty} L(\lambda)^{*} L(\lambda)\|P(d \lambda) x\|^{2}\right\} d t
\end{aligned}
$$

\section{Lévy Flows on Algebras}

Let $X=\left(X(t), t \in \mathbb{R}^{+}\right)$be a Lévy process defined on a suitable probability space $(\Omega, \mathfrak{F}, P)$. For simplicity we will assume that the Lévy measure $v$ of $X$ is finite so that, for each $t \in \mathbb{R}^{+}$we have the Lévy-Itô decomposition

$$
X(t)=m t+\sigma B(t)+\int_{0}^{t+} \int_{\mathbb{R}-\{0\}} x N(d t, d x),
$$

where $m, \sigma \in \mathbb{R}, B=\left(B(t), t \in \mathbb{R}^{+}\right)$is a standard Brownian motion and $N$ is a Poisson random measure on $\mathbb{R}^{+} \times(\mathbb{R}-\{0\})$ which is independent of $B$.

We realise $X$ in $\Gamma\left(L^{2}\left(\mathbb{R}^{+}, \mathfrak{H}_{1}\right)\right)$ as follows (see [Par] p. 152-62). Using the notation of Sect. 5 above, choose $w \in \mathfrak{H}_{1}, x \in \operatorname{Dom}(Y)$ such that, $\|w\|=1, P(\{0\}) w=$ $w$ and $P(\mathbb{R}-\{0\}) x=x$. We fix the Lévy measure $v$ by defining

$$
v(G)=(x, P(G) x), \quad \text { where } G \in \mathscr{B}(\mathbb{R}-\{0\}) .
$$


Now define an adapted process $\hat{X}=\left(\hat{X}(t), t \in \mathbb{R}^{+}\right)$in $\Gamma\left(L^{2}\left(\mathbb{R}^{+}, \mathfrak{G}_{1}\right)\right)$ by

$$
\begin{aligned}
\hat{X}(t)=m t & +i \sigma\left(a\left(\chi_{[0, t)} \otimes w\right)-a^{\dagger}\left(\chi_{[0, t)} \otimes w\right)\right. \\
& +a^{\dagger}\left(\chi_{[0, t)} \otimes Y x\right)+\lambda\left(\chi_{[0, t)} \otimes Y\right)+a\left(\chi_{[0, t)} \otimes Y x\right)+t(x, Y x) .
\end{aligned}
$$

In fact each $\hat{X}(t)$ is essentially self-adjoint on $\mathscr{E}(\mathscr{S})$ and for each $t \in \mathbb{R}^{+}, y \in \mathbb{R}$, we have the Lévy-Khintchine formula $\left\langle\psi(0), \exp \left(i\right.\right.$ y $\left.\left.\hat{X}(t)^{c}\right) \psi(0)\right\rangle$

$$
=\exp \left\{t\left(\operatorname{im} y-\frac{1}{2} \sigma^{2} y^{2}+\int_{\mathbb{R}-\{0\}}\left(e^{2 x y}-1\right) v(d x)\right)\right\}
$$

(where ${ }^{c}$ denotes closure), so that $\hat{X}$ is indeed an operator-valued realisation of $X$. We observe that we have the following decomposition of $\hat{X}$ :

$$
\begin{gathered}
\hat{X}(t)=m t+\int_{0}^{t} i \sigma\left(d A_{w}(s)-d A_{w}^{\dagger}(s)\right) \\
+\int_{-\infty}^{\infty} \int_{0}^{t} \lambda\left\{A_{x}^{\dagger}(d s, P(\lambda))+\Lambda(d s, P(d \lambda))+A_{x}(d s, P(d \lambda))\right. \\
\quad+(x, P(d \lambda) x) d s\} .
\end{gathered}
$$

Comparing (6.3) with (6.1), leads us to introduce the notation

and

$$
d \hat{B}(t)=i\left(d A_{w}(t)-d A_{w}^{\dagger}(t)\right)
$$

$$
\hat{N}(d t, d \lambda)=A_{x}^{\dagger}(d t, P(\lambda))+\Lambda(d t, P(d \lambda))+A_{x}(d t, P(d \lambda))+(x, P(d \lambda) x) d t .
$$

We will construct quantum stochastic flows on $a^{*}$-subalgebra $A$ of $B\left(\mathfrak{H}_{0}\right)$ which are driven by $\hat{X}$. To this end, let $L$ be a bounded skew-adjoint operator on $\mathfrak{S}_{0}, H$ a bounded self-adjoint operator on $\mathfrak{S}_{0}$ and $T$ a not necessarily bounded self-adjoint operator in $\mathfrak{H}_{0}$. Consider the strongly continuous one parameter unitary group $\{W(\lambda), \lambda \in \mathbb{R}\}$ on $\mathfrak{H}_{0}$, where each $W(\lambda)=\exp (i \lambda T)$. If we take each $L(\lambda)=W(\lambda)-I$ and $H_{0}=m H+(x, \sin (T \otimes Y) x)$ then the conditions (i) to (iii) given at the beginning of Sect. 5 are all clearly satisfied. Hence by Theorem 5.4, we can assert the existence of a unique unitary operator valued solution to the SDE

$$
\begin{aligned}
U(t)=I+\int_{0}^{t} U(s)( & \left(\operatorname{im} H+\frac{1}{2} \sigma^{2} L^{2}\right) d s+\int_{0}^{t} \sigma U(s) L d \hat{B}(s) \\
& \left.+\int_{-\infty}^{\infty} \int_{0}^{t} U(s)(W(\lambda)-I) \hat{N}(d s, d \lambda)\right)
\end{aligned}
$$

(c.f. App[2]).

If we now assume that $[H,],.[L,$.$] and each W(\lambda)(). W(\lambda)^{*}$ preserve $\mathscr{A}$, we obtain a quantum stochastic flow $J=\left(j_{t}, t \in \mathbb{R}^{+}\right)$on $\mathscr{A}$ by the prescription (5.2).

A slight extension of (5.3) then yields

$$
\begin{aligned}
j_{t}(a)= & a+\int_{0}^{t}\left\{\operatorname{im} j_{s}([H, a])+\frac{1}{2} \sigma^{2} j_{s}([L,[L, a]])\right\} d s \\
& +\sigma \int_{0}^{t} j_{s}([L, a]) d \hat{B}(s)+\int_{-\infty}^{\infty} \int_{0}^{t} j_{s}\left(W(\lambda) a W(\lambda)^{*}-a\right) \hat{N}(d s, d \lambda),
\end{aligned}
$$


and we have a dilation of the semigroup with generator

$$
\delta(a)=\operatorname{im}[H, a]+\frac{1}{2} \sigma^{2}[L,[L, a]]+\int_{-\infty}^{\infty}\left(W(\lambda) a W(\lambda)^{*}-a\right) v(d \lambda) .
$$

We regard (6.5) as a generalisation to arbitrary ${ }^{*}$-subalgebras of $B\left(\mathfrak{H}_{0}\right)$ of the Lévy flows of diffeomorphisms of manifolds constructed in [ApKu]. In the latter case $\mathfrak{H}_{0}$ is the intrinsic Hilbert space of a manifold and $\mathscr{A}$ the algebra of smooth functions with compact support (see the discussions in [App 2] and [Mey]). An obvious generalisation of the results of this section would be to consider arbitrary Lévy measures. This requires us to extend the results of Sect. 5 to construct unitary operator valued solutions of SDE's driven by infinite series of quantum spectral stochastic integrals. The details will appear elsewhere.

We observe that the methods developed herein can also be applied to obtain a larger class of the "quantum Lévy flows" described in [App 1].

Acknowledgement. It is a great pleasure to thank Robin Hudson for valuable comments on an earlier draft of this paper and Martin Brooks for correcting a number of errors.

\section{References}

[App 1] Applebaum, D.: Deformations of Cocycles, Quantum Lévy Processes and Quantum Stochastic Flows. Rep. Math. Phys. 32, 117-32 (1993)

[App 2] Applebaum, D.: (i) Unitary Actions of Lévy Flows of Diffeomorphisms, J. Multivariate Anal. 49, 266-77 (1994), (ii) An Operator Theoretic Approach to Stochastic Flows on Manifolds, Seminaire de Probabilités XXVI, Springer LNM 1526, 514-33 (1992)

[ApKu] Applebaum, D., Kunita, H.: Lévy Flows on Manifolds and Lévy Processes on Lie Groups. J. Math. Kyoto Univ. 33, 1103-1123 (1993)

[Bel] Belavkin, V.P.: Quantum Continual Measurements and a Posteriori Collapse on CCR. Commun. Math. Phys. 146, 611-35 (1992)

[Ev] Evans, M.P.: Quantum Diffusions. University of Nottingham Ph. D. thesis (1988)

[HuPa 1] Hudson, R.L., Parthasarathy, K.R.: Quantum Itô's Formula and Stochastic Evolution. Commun. Math. Phys. 93, 301-23 (1984)

[HuPa 2] Hudson, R.L., Parthasarathy, K.R.: Stochastic Dilations of Uniformly Continuous Completely Positive Semigroups. Acta. Appl. Math. 2, 353-98 (1984)

[Hud] Hudson, R.L.: Algebraic Theory of Quantum Diffusions. Springer LNM 1325, 113-25 (1988)

[Lin] Lindblad, G.: On the Generators of Quantum Dynamical Semigroups. Commun. Math. Phys. 48, 119-30 (1976)

[Mey] Meyer, P.A.: Quantum Probability for Probabilists. Springer LNM 1538, Berlin, Heidelberg, New York: Springer 1993

[PaSi] Parthasarathy, K.R., Sinha, K.B.: (i) Stochastic Integral Representations of Bounded Quantum Martingales in Fock Space. J. Funct. Anal. 67, 126-51 (1986); (ii) Representation of a Class of Quantum Martingales II. Springer LNM 1303, 232-51 (1988)

[Par] Parthasarathy, K.R.: An Introduction to Quantum Stochastic Calculus. Basel: Birkhäuser 1992 